\title{
A NEW PLANAR BEZIER-LIKE SPIRAL DEFINED BY EXPONENTIAL FUNCTIONS AND TRANSITION CURVES
}

\author{
ASLI AYAR AND BAYRAM ŞAHIN
}

\begin{abstract}
In this paper, we first obtain a new spiral curve by using the curve introduced by Zhu-Han [14] under certain restrictions. Then by using this spiral curve, we construct five transition curves which are from a circle to a line, from a circle to a circle with the $C$-shaped curves, from a circle to a circle with the $S$-shaped curves, from a line to a line and from a circle to a circle, one circle inside the other.
\end{abstract}

\section{Introduction}

Curves and surfaces are used in computer-aided design (CAD) and manufacturing (CAM). Therefore finding or interpolating new curves and surfaces is an important research area of CAD and CAM. The most known and commonly used in CAD/CAM curves are Bezier curves and B-spline curves [4]. These curves are related to their control points and have a control polygon. For the modifications of the curve form, designers can use this control polygon. Since Bezier curves are polynomial such property creates some difficulties for a designer. For instance, if the designer wants to change the curve in a small area, this affects the entire curve. This is an unwanted situation. Therefore, many authors seek new curves with the shape parameters. By using shape parameters the designer can control the curve without changing control points.

Although Bezier curves have been used for design, these curves are not useful for certain cases. For instance, they are not appropriate for representing closed curves. Considering curves with shape parameters, researchers have started to modify representation of curves [1], [2], [3], [6], [10] and [12]. Thus, rational curves, trigonometric curves and Bezier like curves with exponential functions are obtained.

The Bezier like curves with exponential functions, introduced by Zhu and Han in [14]. Basis functions of these curves were obtained by adding two exponential functions to Bernstein basis functions. These two exponential functions have two shape parameters, $\lambda$ and $\mu$. These shape parameters are called tension parameters. Then by incorporating control points with these basis functions, the curve is defined and is called $\lambda \mu$-Bezier like curve with two shape parameters. $\lambda \mu$-Bezier like curve with two shape parameters are closer control polygon than other Bezier

2010 Mathematics Subject Classification. 68U7, 65D15.

Key words and phrases. Bezier curve, Bezier-like curve, Spiral curve, Transition curves. 
like curves. Since these curves are defined with appropriate parameters, it is possible for the designer to make the desired adjustments. More information about these curves can be found in [14].

Also, spirals, which are commonly used in CAD and CAM, are very important for technological design. Because their curvature varies monotonically with their arc length. They don't have cusps, loops or inflection points by definition. Consequently, the spirals are used in many applications such as satellite orbit, railway, highway or robotic design. In [11], the authors found a spiral Bezier curve and then they constructed many transition curves by using this spiral. Later they extended their result for general case [12], [13], see also [1], [5], [7] and [8].

In this paper, we obtain certain conditions for a $\lambda \mu$-Bezier like curve with two shape parameters to be a spiral. After obtaining spiral conditions for this curve, we construct transitions curves by using this spiral, i.e, from a line to a circle transition, from a circle to a circle transition with the $\mathrm{C}$-shaped curves, from a circle to a circle transition with the S-shaped curves, from a line to a line transition. Since these curves are defined with appropriate parameters, the conditions for such curves to be spiral and the construction of transition curves by using these curves can be possible by choosing the appropriate parameters without changing the control points.

\section{Background, notations and conventions}

For any vector $\mathbf{U}$, its parametric form is $\mathbf{U}=\left(U_{x}, U_{y}\right)$. The norm of vector $\mathbf{U}$ is formulated as $\|\mathbf{U}\|=\sqrt{U_{x}^{2}+U_{y}^{2}}$. Positive angles are measured anti-clockwise. The dot product of two vectors, $\mathbf{U}$ and $\mathbf{V}$ is $\mathbf{U} . \mathbf{V}=U_{x} V_{x}+U_{y} V_{y}$ and the cross product of these two vectors is defined as $\mathbf{U} \times \mathbf{V}=\|\mathbf{U}\| \cdot\|\mathbf{V}\| \cdot \sin \theta$ where the angle $\theta$ is a positive angle. The derivative of function $\mathbf{f}$ is denoted by $\mathbf{f}^{\prime}$.

The signed curvature of a Bezier like curve $\mathbf{f}(t)$ is defined by [9]

$$
\kappa(t)=\frac{\left\|\mathbf{f}^{\prime}(t) \times \mathbf{f}^{\prime \prime}(t)\right\|}{\left\|\mathbf{f}^{\prime}(t)\right\|^{3}} .
$$

The radius of curvature $r$ is given by $r=\frac{1}{\kappa}$. If $\mathbf{t}$ is the unit tangent vector to $\mathbf{f}(t)$ at $t$, then the orientation of the unit normal vector $\mathbf{n}$ to $\mathbf{f}(t)$ at $t$, is such that the angle is anti-clockwise from $\mathbf{t}$ to $\mathbf{n}$ is $\pi / 2$.

We now define $\lambda \mu$-Bezier like basis functions with two shape parameters and discuss some of their properties.

Definition 2.1. [14] For $t \in[0,1], \lambda \mu$-Bezier like basis functions with two shape parameters $\lambda, \mu \in[0, \infty)$ are defined as:

$$
\begin{aligned}
& A_{0}(t ; \lambda)=(1-t)^{3} e^{-\lambda t} \\
& A_{1}(t ; \lambda)=(1-t)^{2}\left[1+2 t-(1-t) e^{-\lambda t}\right], \\
& A_{2}(t ; \mu)=t^{2}\left[3-2 t-t e^{-\mu(1-t)}\right] \\
& A_{3}(t ; \mu)=t^{3} e^{-\mu(1-t)} \\
& A_{0}(t ; \lambda)+A_{1}(t ; \lambda)+A_{2}(t ; \mu)+A_{3}(t ; \mu)=1 .
\end{aligned}
$$


For $\lambda, \mu=0$, the $\lambda \mu$-Bezier like basis functions are linear cubic Bernstein basis functions.

$$
\begin{aligned}
& A_{0}(t ; 0)=(1-t)^{3} e^{-0 t}=(1-t)^{3} \\
& A_{1}(t ; 0)=(1-t)^{2}\left[1+2 t-(1-t) e^{-0 t}\right]=(1-t)^{2}[3 t] \\
& A_{2}(t ; 0)=t^{2}\left[3-2 t-t e^{-0(1-t)}\right]=t^{2}[3-2 t-t]=t^{2} 3[1-t], \\
& A_{3}(t ; 0)=t^{3} e^{-0(1-t)}=t^{3}
\end{aligned}
$$

We now construct the $\lambda \mu$-Bezier like curve with two shape parameters as follows:

Definition 2.2. [14] Given the control points $\mathbf{P}_{i}(i=0,1,2,3)$ in $E^{2}$, we define the $\lambda \mu$-Bezier like curve with two shape parameters as:

$$
\begin{aligned}
\mathbf{f}(t ; \lambda, \mu) & =\sum_{i=0}^{3} A_{i}(t ; \lambda, \mu) \mathbf{P}_{i} \\
& =A_{0}(t ; \lambda) \mathbf{P}_{0}+A_{1}(t ; \lambda) \mathbf{P}_{1}+A_{2}(t ; \mu) \mathbf{P}_{2}+A_{3}(t ; \mu) \mathbf{P}_{3} \\
& t \in[0,1], \quad \lambda, \mu \in[0, \infty) .
\end{aligned}
$$

The curve defined by (2.5) satisfies some geometric properties which can be obtained easily from the properties of the basis functions.

$$
\begin{array}{ll}
A_{0}(0 ; \lambda)=1, & A_{0}(1 ; \lambda)=0, \\
A_{1}(0 ; \lambda)=0, & A_{1}(1 ; \lambda)=0, \\
A_{2}(0 ; \mu)=0, & A_{2}(1 ; \mu)=0, \\
A_{3}(0 ; \mu)=0, & A_{3}(1 ; \mu)=1 .
\end{array}
$$

Hermite curves are defined by two points and two tangent vectors. Here,

$$
\begin{aligned}
& \mathbf{f}(0 ; \lambda, \mu)=\mathbf{P}_{0}, \\
& \mathbf{f}(1 ; \lambda, \mu)=\mathbf{P}_{3} .
\end{aligned}
$$

It is easy to see that the curve provides Hermite conditions. On the other hand, by direct computations we have

$$
\begin{gathered}
A_{0}{ }^{\prime}(t ; \lambda)=e^{-\lambda t}(1-t)^{2}[-3-(1-t) \lambda] \\
A_{1}{ }^{\prime}(t ; \lambda)=e^{-\lambda t}(1-t)\left[3+\lambda+t^{2} \lambda-t\left(3+6 e^{\lambda t}+2 \lambda\right)\right], \\
A_{2}{ }^{\prime}(t ; \mu)=e^{-\mu}\left[6 e^{\mu}(1-t)-e^{\mu t} t(3+\mu t)\right] \\
A_{3}{ }^{\prime}(t ; \mu)=e^{-\mu(1-t)} t^{2}(3+\mu t), \\
A_{0}^{\prime \prime}(t ; \lambda)=e^{-\lambda t}(1-t)\left[6+6(1-t) \lambda+(1-t)^{2} \lambda^{2}\right], \\
A_{1}^{\prime \prime}(t ; \lambda)=e^{-\lambda t}\left[6 e^{\lambda t}(-1+2 t)-(1-t)\left(6+6(1-t) \lambda+(1-t)^{2} \lambda^{2}\right)\right], \\
A_{2}^{\prime \prime}(t ; \mu)=e^{-\mu}\left[e^{\mu}(6-12 t)-e^{\mu t} t\left(6+6 \mu t+\mu^{2} t^{2}\right)\right], \\
A_{3}^{\prime \prime}(t ; \mu)=e^{-\mu(1-t)} t\left(6+6 \mu t+\mu^{2} t^{2}\right), \\
A_{0}{ }^{\prime \prime \prime}(t ; \lambda)=e^{-\lambda t}\left[-6-18(1-t) \lambda-9(1-t)^{2} \lambda^{2}-(1-t)^{3} \lambda^{3}\right], \\
A_{1}^{\prime \prime \prime}(t ; \lambda)=e^{-\lambda t}\left[6+12 e^{\lambda t}+18(1-t) \lambda+9(1-t)^{2} \lambda^{2}+(1-t)^{3} \lambda^{3}\right], \\
A_{2}{ }^{\prime \prime \prime}(t ; \mu)=e^{-\mu}\left[-12 e^{\mu}-e^{\mu t} t\left(6+18 \mu t+9 \mu^{2} t^{2}+t^{3} \mu^{3}\right)\right], \\
A_{3}{ }^{\prime \prime \prime}(t ; \mu)=e^{-\mu(1-t)}\left(6+18 \mu t+9 \mu^{2} t^{2}+t^{3} \mu^{3}\right) .
\end{gathered}
$$


Hence, we have

$$
\begin{aligned}
& \mathbf{f}^{\prime}(0 ; \lambda, \mu)=(\lambda+3)\left(\mathbf{P}_{1}-\mathbf{P}_{0}\right) \\
& \mathbf{f}^{\prime}(1 ; \lambda, \mu)=(\mu+3)\left(\mathbf{P}_{3}-\mathbf{P}_{2}\right) \\
& \mathbf{f}^{\prime \prime}(0 ; \lambda, \mu)=\left(\lambda^{2}+6 \lambda+6\right)\left(\mathbf{P}_{0}-\mathbf{P}_{1}\right)+6\left(\mathbf{P}_{2}-\mathbf{P}_{1}\right) \\
& \mathbf{f}^{\prime \prime}(1 ; \lambda, \mu)=\left(\mu^{2}+6 \mu+6\right)\left(\mathbf{P}_{3}-\mathbf{P}_{2}\right)+6\left(\mathbf{P}_{1}-\mathbf{P}_{2}\right) .
\end{aligned}
$$

Therefore, the tangent vector at the starting point is parallel to $\left(\mathbf{P}_{1}-\mathbf{P}_{0}\right)$ and the tangent vector at the endpoint is parallel to $\left(\mathbf{P}_{3}-\mathbf{P}_{2}\right)$. Then, we say that curve provides other Hermite conditions and this curve is defined by two points and two tangent vectors.

\section{3. $\lambda \mu$-Bezier like spiral with two shape parameters}

In this section, we are going to find a new spiral under certain conditions for $\lambda \mu$-Bezier like curve given in Definition 2.1.

Theorem 3.1. Let beginning and ending unit tangent vectors be $\mathbf{t}_{0}$ and $\mathbf{t}_{1}$ of $\lambda \mu-$ Bezier like curve with two shape parameters respectively. The beginning point of curve is $\mathbf{P}_{0}$ and it is given. The ending curvature value is $c>0$. We define the $\lambda \mu-$ Bezier like curve with two shape parameters as

$$
\begin{gathered}
\mathbf{f}(t ; \lambda, \mu)=A_{0}(t ; \lambda) \mathbf{P}_{0}+A_{1}(t ; \lambda) \mathbf{P}_{1}+A_{2}(t ; \mu) \mathbf{P}_{2}+A_{3}(t ; \mu) \mathbf{P}_{3} \\
t \in[0,1], \quad \lambda \in(1, \infty), \quad \mu \in(3, \infty)
\end{gathered}
$$

with

$$
\begin{aligned}
& \mathbf{P}_{1}=\mathbf{P}_{0}+a \mathbf{t}_{\mathbf{0}}, \\
& \mathbf{P}_{2}=\mathbf{P}_{1}+a \mathbf{t}_{\mathbf{0}}=\mathbf{P}_{0}+2 a \mathbf{t}_{0}, \\
& \mathbf{P}_{3}=\mathbf{P}_{2}+b \mathbf{t}_{1}=\mathbf{P}_{0}+2 a \mathbf{t}_{\mathbf{0}}+b \mathbf{t}_{1}
\end{aligned}
$$

for $a, b>0$. Suppose $\theta$ is the positive angle from $\mathbf{t}_{0}$ to $\mathbf{t}_{1}$. The $\lambda \mu$-Bezier like curve is a spiral segment when

$$
a=\frac{e^{-2 \lambda}\left(3+\mu+e^{\lambda}(12+\mu(16+3 \mu))\right)^{2} \sec \theta \tan \theta}{54 c(3+\mu)^{2}}
$$

and

$$
b=\frac{e^{-\lambda}\left(3+\mu+e^{\lambda}(12+\mu(16+3 \mu))\right) \tan \theta}{3 c(3+\mu)^{2}} .
$$

This $\lambda \mu$-Bezier like curve has

$$
\begin{gathered}
\kappa(0)=0, \\
\kappa(1)=c, \kappa^{\prime}(1)=0
\end{gathered}
$$

and $\kappa^{\prime}(t) \neq 0$ for $0 \leq t<1$. Note that, for $\lambda=\mu=0$, we obtain following conditions which Walton and Meek obtained in [11],

$$
a=\frac{25 \sec \theta \tan \theta}{54 c}
$$

and

$$
b=\frac{5 \tan \theta}{9 c} .
$$


Proof. Let $\mathbf{n}_{0}$ be the normal vector at $\mathbf{P}_{0}$. Substitute of $\mathbf{P}_{1}, \mathbf{P}_{2}, \mathbf{P}_{3}$ from (3.1) into (2.5) to obtain

$$
\mathbf{f}(t)=\mathbf{P}_{0}+a\left(A_{1}(t ; \lambda)+2 A_{2}(t ; \mu)+2 A_{3}(t ; \mu)\right) \mathbf{t}_{0}+A_{3}(t ; \mu) b \mathbf{t}_{1} .
$$

Then take the derivative to produce

$$
\begin{aligned}
& \mathbf{f}^{\prime}(t)=\left(A_{1}{ }^{\prime}(t ; \lambda)+2 A_{2}{ }^{\prime}(t ; \mu)+2 A_{3}{ }^{\prime}(t ; \mu)\right) a \mathbf{t}_{\mathbf{0}}+A_{3}{ }^{\prime}(t ; \mu) b \mathbf{t}_{1}, \\
& \mathbf{f}^{\prime \prime}(t)=\left(A_{1}^{\prime \prime}(t ; \lambda)+2 A_{2}^{\prime \prime}(t ; \mu)+2 A_{3}^{\prime \prime}(t ; \mu)\right) a \mathbf{t}+A_{3}^{\prime \prime}(t ; \mu) b \mathbf{t}_{1} .
\end{aligned}
$$

Since $\mathbf{t}_{1}=\cos \theta \mathbf{t}_{0}+\sin \theta \mathbf{n}_{\mathbf{0}}$. Substituting expressions $A_{1}{ }^{\prime}(t ; \lambda), A_{2}{ }^{\prime}(t ; \lambda), A_{3}{ }^{\prime}(t ; \mu)$, $A_{1}{ }^{\prime \prime}(t ; \lambda), A_{2}{ }^{\prime \prime}(t ; \mu), A_{3}{ }^{\prime \prime}(t ; \mu)$ given in (2.8) and (2.9) into (3.9) and (3.10), we get

$$
\begin{aligned}
\mathbf{f}(t)= & \mathbf{P}_{0}+\left\{a\left(A_{1}(t ; \lambda)+2 A_{2}(t ; \mu)+2 A_{3}(t ; \mu)\right)+b \cos \theta A_{3}(t ; \mu)\right\} \mathbf{t}_{0} \\
& +A_{3}(t ; \mu) b \sin \theta \mathbf{n}_{0} \\
\mathbf{f}^{\prime}(t)= & \left\{a\left(A_{1}{ }^{\prime}(t ; \lambda)+2 A_{2}{ }^{\prime}(t ; \mu)+2 A_{3}{ }^{\prime}(t ; \mu)\right)+b \cos \theta A_{3}{ }^{\prime}(t ; \mu)\right\} \mathbf{t}_{0} \\
& +A_{3}{ }^{\prime}(t ; \mu) b \sin \theta \mathbf{n}_{0} \\
= & x^{\prime}(t) \mathbf{t}_{0}+y^{\prime}(t) \mathbf{n}_{0}
\end{aligned}
$$

and

$$
\begin{aligned}
\mathbf{f}^{\prime \prime}(t)= & \left\{a\left(A_{1}^{\prime \prime}(t ; \lambda)+2 A_{2}{ }^{\prime \prime}(t ; \mu)+2 A_{3}{ }^{\prime \prime}(t ; \mu)\right)+b \cos \theta A_{3}{ }^{\prime \prime}(t ; \mu)\right\} \mathbf{t}_{0} \\
& +A_{3}^{\prime \prime}(t ; \mu) b \sin \theta \mathbf{n}_{0} \\
= & x^{\prime \prime}(t) \mathbf{t}_{\mathbf{0}}+y^{\prime \prime}(t) \mathbf{n}_{0},
\end{aligned}
$$

where

$$
\begin{aligned}
& x^{\prime}(t)=a\left(A_{1}{ }^{\prime}(t ; \lambda)+2 A_{2}{ }^{\prime}(t ; \mu)+2 A_{3}{ }^{\prime}(t ; \mu)\right)+b \cos \theta A_{3}{ }^{\prime}(t ; \mu), \\
& y^{\prime}(t)=A_{3}{ }^{\prime}(t ; \mu) b \sin \theta \\
& x^{\prime \prime}(t)=a\left(A_{1}^{\prime \prime}(t ; \lambda)+2 A_{2}^{\prime \prime}(t ; \mu)+2 A_{3}^{\prime \prime}(t ; \mu)\right)+b \cos \theta A_{3}^{\prime \prime}(t ; \mu), \\
& y^{\prime \prime}(t)=A_{3}^{\prime \prime}(t ; \mu) b \sin \theta \\
& x^{\prime \prime \prime}(t)=a\left(A_{1}^{\prime \prime \prime}(t ; \lambda)+2 A_{2}^{\prime \prime \prime}(t ; \mu)+2 A_{3}^{\prime \prime \prime}(t ; \mu)\right)+b \cos \theta A_{3}^{\prime \prime \prime}(t ; \mu), \\
& y^{\prime \prime \prime}(t)=A_{3}{ }^{\prime \prime \prime}(t ; \mu) b \sin \theta .
\end{aligned}
$$

The curvature and its derivative are given by

$$
\kappa(t)=\frac{\left\{x^{\prime}(t) y^{\prime \prime}(t)-x^{\prime \prime}(t) y^{\prime}(t)\right\}}{\left\{\left(x^{\prime}(t)\right)^{2}+\left(y^{\prime}(t)\right)^{2}\right\}^{3 / 2}}
$$

and

$$
\begin{gathered}
\kappa^{\prime}(t)=\frac{\left(\mathbf{f}^{\prime}(t) \times \mathbf{f}^{\prime \prime \prime}(t)\right)\left(\mathbf{f}^{\prime}(t) \mathbf{f}^{\prime}(t)\right)-3\left(\mathbf{f}^{\prime}(t) \times \mathbf{f}^{\prime \prime}(t)\right)\left(\mathbf{f}^{\prime}(t) \mathbf{f}^{\prime \prime}(t)\right)}{\left\|\mathbf{f}^{\prime}(t)\right\|^{5}}, \\
\kappa^{\prime}(t)=\frac{A(t)}{\left\|\mathbf{f}^{\prime}(t)\right\|^{5}}
\end{gathered}
$$


respectively, where

$$
\begin{aligned}
A(t) & =\left(\mathbf{f}^{\prime}(t) \times \mathbf{f}^{\prime \prime \prime}(t)\right)\left(\mathbf{f}^{\prime}(t) \mathbf{f}^{\prime}(t)\right)-3\left(\mathbf{f}^{\prime}(t) \times \mathbf{f}^{\prime \prime}(t)\right)\left(\mathbf{f}^{\prime}(t) \mathbf{f}^{\prime \prime}(t)\right) \\
& =\left(x^{\prime}(t) y^{\prime \prime \prime}(t)-x^{\prime \prime \prime}(t) y^{\prime}(t)\right)\left(\left(x^{\prime}(t)\right)^{2}+\left(y^{\prime}(t)\right)^{2}\right) \\
& -3\left(x^{\prime}(t) y^{\prime \prime}(t)-x^{\prime \prime}(t) y^{\prime}(t)\right)\left(x^{\prime}(t) x^{\prime \prime}(t)+y^{\prime}(t) y^{\prime \prime}(t)\right) .
\end{aligned}
$$

From (3.16), we can say that $A(t)$ affects its sign. On the other hand, if $\kappa^{\prime}(t)$ has a root at a point, $\kappa^{\prime}(t)=0$ at that point. From (3.16), if $\kappa^{\prime}(t)=0$ then $A(t)$ must be equal to zero. For this reason, we research the conditions for $A(t) \neq 0$ in $[0,1]$. Set

$$
A(t)=a(t) b(t)-3 c(t) d(t)
$$

where

$$
\begin{aligned}
& a(t)=\left(\mathbf{f}^{\prime}(t) \times \mathbf{f}^{\prime \prime \prime}(t)\right)=x^{\prime}(t) y^{\prime \prime \prime}(t)-x^{\prime \prime \prime}(t) y^{\prime}(t), \\
& b(t)=\left(\mathbf{f}^{\prime}(t) \mathbf{f}^{\prime}(t)\right)=\left(x^{\prime}(t)\right)^{2}+\left(y^{\prime}(t)\right)^{2} \\
& c(t)=\left(\mathbf{f}^{\prime}(t) \times \mathbf{f}^{\prime \prime}(t)\right)=x^{\prime}(t) y^{\prime \prime}(t)-x^{\prime \prime}(t) y^{\prime}(t), \\
& d(t)=\left(\mathbf{f}^{\prime}(t) \mathbf{f}^{\prime \prime}(t)\right)=x^{\prime}(t) x^{\prime \prime}(t)+y^{\prime}(t) y^{\prime \prime}(t) .
\end{aligned}
$$

Therefore we have

$$
\begin{array}{rlrl}
a(t) & =x^{\prime}(t) y^{\prime \prime \prime}(t)-x^{\prime \prime \prime}(t) y^{\prime}(t) & =a_{1}(t)-a_{2}(t), \\
b(t) & =\left(x^{\prime}(t)\right)^{2}+\left(y^{\prime}(t)\right)^{2} & & =b_{1}(t)+b_{2}(t), \\
c(t) & =x^{\prime}(t) y^{\prime \prime}(t)-x^{\prime \prime}(t) y^{\prime}(t) & & =c_{1}(t)-c_{2}(t), \\
d(t) & =x^{\prime}(t) x^{\prime \prime}(t)+y^{\prime}(t) y^{\prime \prime}(t) & & =d_{1}(t)+d_{2}(t)
\end{array}
$$

where

$$
\begin{aligned}
a_{1}(t) & =x^{\prime}(t) y^{\prime \prime \prime}(t), & & a_{2}(t)=x^{\prime \prime \prime}(t) y^{\prime}(t) \\
b_{1}(t) & =\left(x^{\prime}(t)\right)^{2}, & & b_{2}(t)=\left(y^{\prime}(t)\right)^{2} \\
c_{1}(t) & =x^{\prime}(t) y^{\prime \prime}(t), & & c_{2}(t)=x^{\prime \prime}(t) y^{\prime}(t) \\
d_{1}(t) & =x^{\prime}(t) x^{\prime \prime}(t), & & d_{2}(t)=y^{\prime}(t) y^{\prime \prime}(t)
\end{aligned}
$$

Then by direct computations we obtain

$$
\begin{aligned}
a_{1}(t)= & \frac{e^{-3 \lambda-(1-t) \mu}(6+\mu t(3+t \mu)(6+t \mu))\left(3+\mu+e^{\lambda}(12+\mu(16+3 \mu))\right)^{2} \sin \theta \tan \theta}{162 c^{2}(3+\mu)^{4}} \\
& {\left[\left(18 e^{\lambda-(1-t) \mu} t^{2}(3+t \mu) \sin \theta\right)+e^{-t \lambda}(1-t) \sec \theta \tan \theta\right.} \\
& \left.\left(3+\lambda-t\left(3-6 e^{t \lambda}-(t-2) \lambda\right)\right)\left(3+\mu+e^{\lambda}(12+\mu(16+3 \mu))\right)\right], \\
a_{2}(t)= & \frac{e^{-(3+t) \lambda-(1-t) \mu} t^{2}(3+t \mu)\left(3+\mu+e^{\lambda}(12+\mu(16+3 \mu))\right)^{2} \tan ^{3} \theta}{162 c^{2}(3+\mu)^{4}} \\
& {\left[6-12 e^{\lambda . t}+(1-t) \lambda(6+(1-t) \lambda)\right.} \\
& (3+(1-t) \lambda)\left(3+\mu+e^{\lambda}(12+\mu(16+3 \mu))\right)+18 e^{\lambda(1+t)-(1-t) \mu} \\
& \left.\left(6+\mu t(3+t \mu)(6+t \mu)(6+t \mu) \cos ^{2} \theta\right)\right]
\end{aligned}
$$




$$
\begin{aligned}
& b_{1}(t)= \frac{e^{-8 \lambda}}{2916 c^{2}(3+\mu)^{4}}\left(3+\mu+e^{\lambda}(12+\mu(16+3 \mu))\right)^{2} \\
& {\left[-18 e^{\lambda-(1-t) \mu} t^{2}(3+t \mu) \sin \theta-e^{-t \lambda}(1-t)\right.} \\
&\left(3+\lambda-t\left(3-6 e^{t \lambda}-(t-2) \lambda\right)\right) \\
&\left.\left(3+\mu+e^{\lambda}(12+\mu(16+3 \mu) \sec \theta \tan \theta)\right)\right]^{2}, \\
& b_{2}(t)= \frac{e^{-2 \lambda-2(1-t) \mu} t^{4}}{9 c^{2}(3+\mu)^{4}}(3+t \mu)^{2}\left(3+\mu+e^{\lambda}(12+\mu(16+3 \mu))\right)^{2} \sin ^{2} \theta \tan ^{2} \theta, \\
& c_{1}(t) \quad=\frac{e^{-3 \lambda-(1-t) \mu} t}{162 c^{2}(3+\mu)^{4}}(6+\mu t(6+t \mu))\left(3+\mu+e^{\lambda}(12+\mu(16+3 \mu))\right)^{2} \sin ^{2} \theta \\
& \tan \theta\left[18 e^{\lambda-(1-t) \mu} t^{2}(3+t \mu) \sin \theta-e^{-t \lambda}(1-t)\right. \\
&\left(3+\lambda-t\left(3-6 e^{t \lambda}-(t-2) \lambda\right)\right) \\
&\left.\left(3+\mu+e^{\lambda}(12+\mu(16+3 \mu))\right) \sec \theta \tan \theta\right]
\end{aligned}
$$$$
c_{2}(t)=\frac{e^{-3 \lambda-(1-t) \mu} t^{2}(3+t \mu)\left(3+\mu+e^{\lambda}(12+\mu(16+3 \mu))\right)^{2} \tan ^{3} \theta}{162 c^{2}(3+\mu)^{4}}
$$$$
\left[18 e^{\lambda-(1-t) \mu} t(6+\mu t(6+t \mu)) \sin \theta+e^{-t \lambda}\right.
$$$$
\left(6 e^{t \lambda}(1-2 t)-(1-t)(6+(1-t) \lambda(6+\lambda-t \lambda))\right)
$$$$
\left.\left(3+\mu+e^{\lambda}(12+\mu(16+3 \mu))\right) \sec \theta \tan \theta\right],
$$

$$
\begin{aligned}
d_{1}(t)= & \frac{e^{-4 \lambda}\left(3+\mu+e^{\lambda}(12+\mu(16+3 \mu))\right)^{2} \sin \theta \tan \theta}{2916 c^{2}(3+\mu)^{4}} \\
& {\left[18 e^{\lambda-(1-t) \mu} t^{2}(3+\mu t) \sin \theta+e^{-t \lambda}(1-t)\right.} \\
& \left(3+\lambda-t\left(3-6 e^{t \lambda}-(t-2) \lambda\right)\right) \\
& \left(3+\mu+e^{\lambda}(12+\mu(16+3 \mu)) \sec \theta \tan \theta\right. \\
& \left(18 e^{\lambda-(1-t) \mu} t(6+\mu t(6+t \mu)) \sin \theta+e^{-t \lambda}\right. \\
& \left.\left(6 e^{t \lambda}(1-2 t)-(1-t) 3(6+(1-t) \lambda(6+\lambda-t \lambda))\right)\right) \\
& \left.\left(3+\mu+e^{\lambda}(12+\mu(16+3 \mu) \sec \theta \tan \theta)\right)\right]
\end{aligned}
$$

and

$$
\begin{aligned}
& d_{2}(t)=\frac{e^{-2 \lambda-2(1-t) \mu} t^{3}}{9 c^{2}(3+\mu)^{4}}(3+t \mu)(6+\mu t(6+t \mu)) \\
& {\left[\left(3+\mu+e^{\lambda}(12+\mu(16+3 \mu))\right)\right]^{2} \sin ^{2} \theta \tan ^{2} \theta . }
\end{aligned}
$$




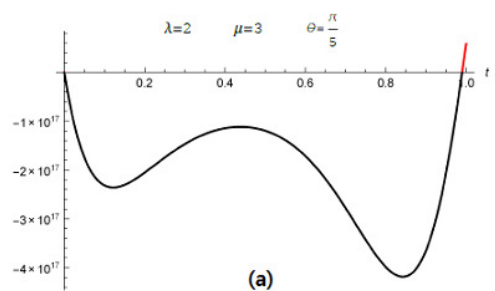

(a)

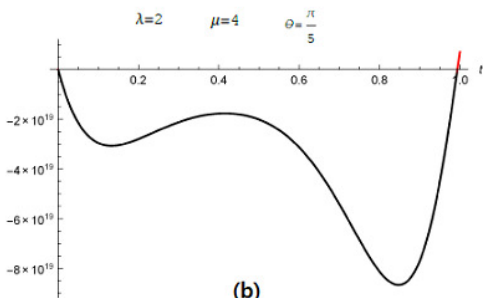

(b)

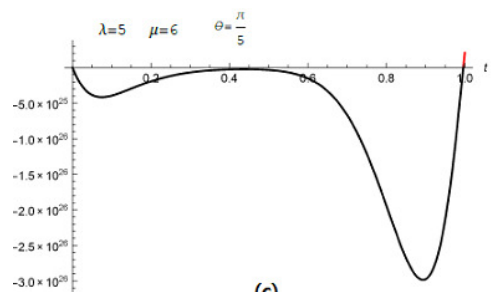

(c)

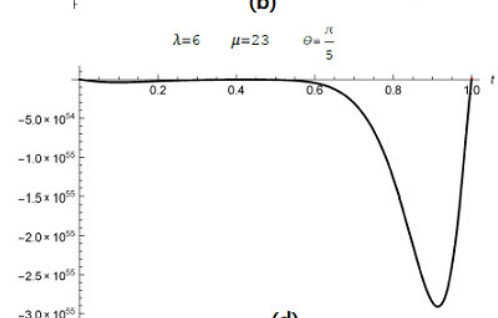

(d)

FiguRE 1. Graphs of derivative of curvature functions

If $t=0$, from (3.13), it follows

$$
\begin{array}{ll}
x^{\prime}(0)=a(3+\lambda), & y^{\prime}(0)=0, \\
x^{\prime \prime}(0)=a\left(-6-6 \lambda-\lambda^{2}\right), & y^{\prime \prime}(0)=0, \\
x^{\prime \prime \prime}(0)=a\left(6+18 \lambda+9 \lambda^{2}+\lambda^{3}\right)+6 e^{-\mu} b \cos \theta, & y^{\prime \prime \prime}(0)=6 e^{-\mu} b \cos \theta .
\end{array}
$$

Using these equations in (3.17), we get

$$
\begin{aligned}
A(0) & =\left(x^{\prime}(0) y^{\prime \prime \prime}(0)-x^{\prime \prime \prime}(0) y^{\prime}(0)\right)\left(\left(x^{\prime}(0)\right)^{2}+\left(y^{\prime}(0)\right)^{2}\right) \\
& -3\left(x^{\prime}(0) y^{\prime \prime}(0)-x^{\prime \prime}(0) y^{\prime}(0)\right)\left(x^{\prime}(0) x^{\prime \prime}(t)+y^{\prime}(0) y^{\prime \prime}(0)\right) \\
& =\left(a(3+\lambda) 6 e^{-\mu} b \cos \theta\right)(a(3+\lambda))^{2} \\
& =6 a^{3} e^{-\mu} b \cos \theta(3+\lambda)^{3} .
\end{aligned}
$$

From (3.16), the beginning curvature value is

$$
\kappa^{\prime}(0)=\frac{A(0)}{\left\|\mathbf{f}^{\prime}(0)\right\|^{5}}>0 .
$$

(3.4) and (3.5) follows substitution of $t=0$ and $t=1$ into (3.14) and (3.15):

$$
\kappa(0)=0, \quad \kappa(1)=c, \quad \kappa^{\prime}(1)=0 .
$$

It also follows from (3.15), $A(0)>0$, and $\lim _{t \rightarrow \infty} A(t)>0$, hence value of $\kappa^{\prime}(t)$ decreases on $0 \leq t<1$, and when $t=1, \kappa^{\prime}(1)=0$. So that $\kappa^{\prime}(t)$ does not change sign on $0 \leq t<1$. From Figure (1) (a),(b),(c) and (d), for arbitrary value of $c$, $\theta$ and $0<t<1$, if $\lambda \in(1, \infty), \mu \in(3, \infty)$ then $\kappa^{\prime}(t)$ does not change sign on $0 \leq t<1$.

From Figure (2) (a),(b),(c),(d),(e) and (f) if $\lambda \in(-\infty, 1], \quad \mu \in(-\infty, 3]$ then one can see that $\kappa^{\prime}(t)$ changes sign on $0 \leq t<1$. 


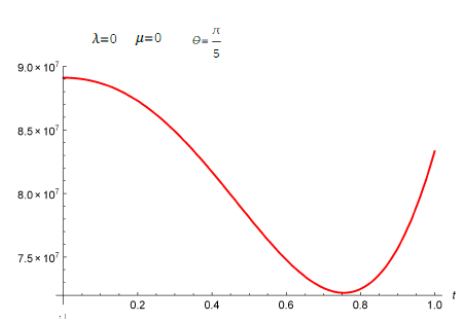

(a)

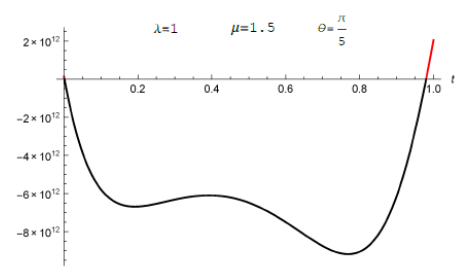

(c)

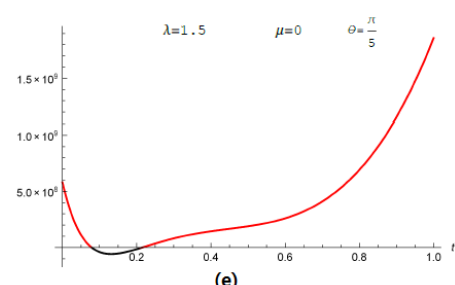

(e)

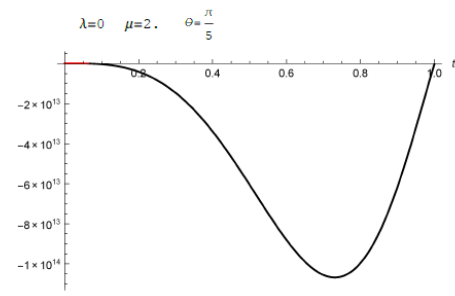

(b)

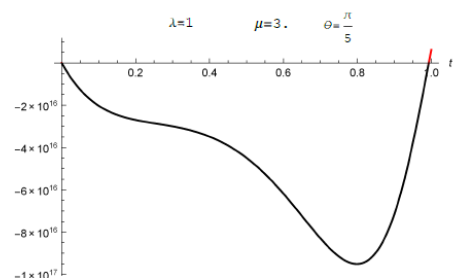

(d)

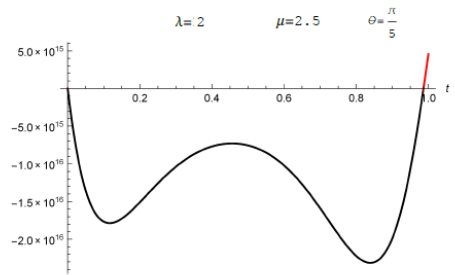

(f)

Figure 2. Graphs of derivative of curvature function

Remark 1. The effect of the shape parameters on the $\lambda \mu$-Bezier like spiral. Obviously, $\lambda$ and $\mu$ shape parameters affect the curve on the control $\mathbf{P}_{1}-\mathbf{P}_{0}$ and $\mathbf{P}_{3}-\mathbf{P}_{2}$, respectively. From [2.2], if $\lambda$ decrease, than $A_{0}(t ; \lambda)$ indecrease for any $t \in[0,1]$. Thus, we can say that the spiral moves in the opposite direction of $\mathbf{P}_{1}-\mathbf{P}_{0}$. If $\lambda$ indecrease, than $A_{0}(t ; \lambda)$ decrease for any $t \in[0,1]$. Thus, we can say that the spiral moves in the same direction of $\mathbf{P}_{1}-\mathbf{P}_{0}$. Similarly, $\mu$ has the same effects on the $\mathbf{P}_{3}-\mathbf{P}_{2}$. Figure 3 shows the effect of $\mu$ shape parameter on the $\lambda \mu$-Bezier like spiral.

We can see that $\lambda$ and $\mu$ shape parameters satisfy local control on the control polygon of the spiral.

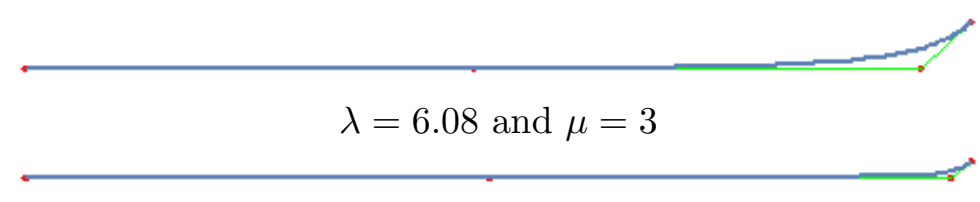

$$
\lambda=6.08 \text { and } \mu=6.14
$$

FiguRE 3. The effect of $\mu$ shape parameter on the $\lambda \mu$-Bezier like spiral.

Remark 2. Comparison between $\lambda \mu$-Bezier like spiral and Bezier spiral. From Fig. [4], we can say the $\lambda \mu$-Bezier like spiral is closer to the control polygon than 
Bezier spiral in [11]. Also, we can see the other advantages of $\lambda \mu$-Bezier like curves in [10].

Figure 4. The yellow curve is a Bezier Spiral, The blue curve is a $\lambda \mu$-Bezier like Spiral

We now construct transition curves by using the spiral which we find in Theorem 3.1.

Theorem 3.2. Let $\mathbf{P}$ be a point and $\mathbf{t}$ be a unit vector of $\mathbf{P}$. Let $\mathbf{d}$ be a line passing through $\mathbf{P}$ and parallel to $\mathbf{t}$. For a circle $M$ with radius $r>0$ centered at $\mathbf{O}$, suppose $\mathbf{t} \times(\mathbf{O}-\mathbf{P})>0$ and $\mathbf{L}=\mathbf{O}-\mathbf{P}$. Let perpendicular distance from $\mathbf{O}$ to $\mathbf{d}$ be $h$.

If $r<h$, then there is a unique $\lambda \mu$-Bezier like spiral with two shape parameters as defined in Theorem (3.1), and shown in Fig. (5), that joins $\mathbf{d}$ to $M$ such that the points of contact are $G^{2}$. The angle from $\mathbf{t}$ to $\mathbf{t}_{1}$ of this $\lambda \mu$-Bezier like spiral satisfies

$$
\frac{r e^{-\lambda}\left(3+\mu+e^{\lambda}(12+\mu(16+3 \mu))\right) \sin \theta \tan \theta}{3(3+\mu)^{2}}-\frac{h}{r}+\cos \theta=0 .
$$

Proof. The unit normal vector at $\mathbf{f}(0 ; \lambda, \mu)$ and $\mathbf{P}$ is given by

$$
\mathbf{n}=\frac{\mathbf{L}-(\mathbf{L} \mathbf{t}) \mathbf{t}}{\|\mathbf{L}-(\mathbf{L} \mathbf{t}) \mathbf{t}\|}
$$

Since $\mathbf{f}(0 ; \lambda, \mu)$ lies on $\mathbf{d}$, and $\mathbf{f}(1 ; \lambda, \mu)$ lies on $M$ it follows that

$$
\mathbf{f}(0 ; \lambda, \mu)=\mathbf{P}+\sigma \mathbf{t}
$$

and

$$
\mathbf{f}(1 ; \lambda, \mu)=\mathbf{P}+(\mathbf{L t}+r \sin \theta) \mathbf{t}+(h-r \cos \theta) \mathbf{n}
$$

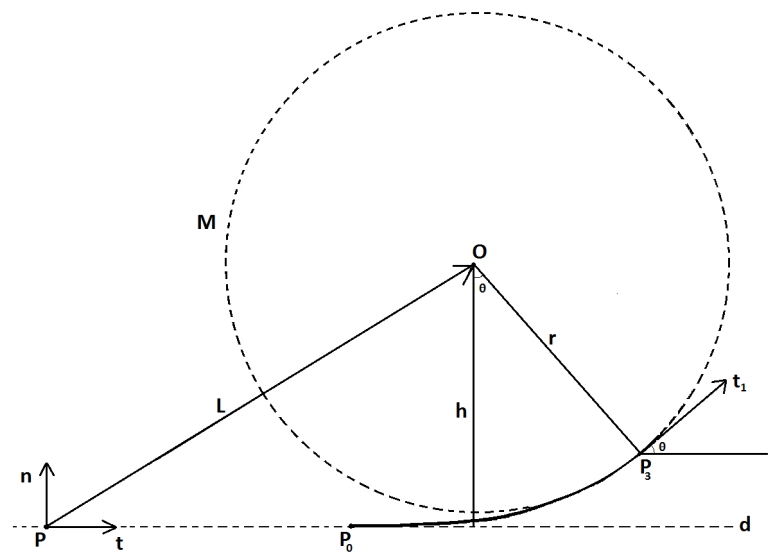

Figure 5. From a line to a circle transition 
From Theorem 3.1 it follows that

$$
\begin{gathered}
\mathbf{f}(0 ; \lambda, \mu)=\mathbf{P}_{0}, \\
\mathbf{f}(1 ; \lambda, \mu)=\mathbf{P}_{3}=\mathbf{P}_{0}+2 a \mathbf{t}+b \mathbf{t}_{1}, \\
a=\frac{e^{-2 \lambda}\left(3+\mu+e^{\lambda}(12+\mu(16+3 \mu))\right)^{2} \sec \theta \tan \theta}{54 c(3+\mu)^{2}}, \\
b=\frac{e^{-\lambda}\left(3+\mu+e^{\lambda}(12+\mu(16+3 \mu))\right) \tan \theta}{3 c(3+\mu)^{2}} .
\end{gathered}
$$

Substitution of these into (3.20) and (3.21), elimination of $\mathbf{P}$, and subsequent rearrangement produce

$$
\begin{aligned}
& \mathbf{f}(0 ; \lambda, \mu)=\mathbf{P}+\sigma \mathbf{t}=\mathbf{P}_{0}, \quad \mathbf{P}=\mathbf{P}_{0}-\sigma \mathbf{t}, \\
& \mathbf{f}(1 ; \lambda, \mu)=\mathbf{P}+(\mathbf{L t}+r \sin \theta) \mathbf{t}+(h-r \cos \theta) \mathbf{n}=\mathbf{P}_{0}+2 a \mathbf{t}+b \mathbf{t}_{1}, \\
& \mathbf{P}=\mathbf{P}_{0}-\sigma \mathbf{t}=\mathbf{P}_{0}+2 a \mathbf{t}+b \mathbf{t}_{1}-(\mathbf{L t}+r \sin \theta) \mathbf{t}-(h-r \cos \theta) \mathbf{n}
\end{aligned}
$$

and

$$
\sigma \mathbf{t}+b \mathbf{t}_{1}-(\mathbf{L t}+r \sin \theta-2 a) \mathbf{t}-(h-r \cos \theta) \mathbf{n}=0 .
$$

(3.18) follows upon taking the dot product of the above with $\mathbf{n}$ and dividing by $r$. Hence we derive

$$
b \sin \theta-(h-r \cos \theta)=0 .
$$

From (3.3) and using $c=1 / r$, we obtain

$$
\frac{r e^{-\lambda}\left(3+\mu+e^{\lambda}(12+\mu(16+3 \mu))\right) \tan \theta}{3(3+\mu)^{2}} \sin \theta-(h-r \cos \theta)=0 .
$$

Set

$$
\begin{aligned}
q(\theta) & =\frac{1}{r}\left(\frac{r e^{-\lambda}\left(3+\mu+e^{\lambda}(12+\mu(16+3 \mu))\right) \tan \theta}{3(3+\mu)^{2}} \sin \theta-(h-r \cos \theta)\right) \\
& =\frac{e^{-\lambda}\left(3+\mu+e^{\lambda}(12+\mu(16+3 \mu)) \sin \theta \tan \theta\right.}{3(3+\mu)^{2}}-\frac{h}{r}+\cos \theta
\end{aligned}
$$

Now, for $\theta=0$ we have

$$
q(0)=1-\frac{h}{r}
$$

From the requirement $r<h \Rightarrow 1<\frac{h}{r}$, it follows

$$
q(0)=1-\frac{h}{r}<0
$$

For $\theta=\pi / 2$ we obtain

$$
q(\pi / 2)=\infty>0
$$

and for derivative of $q(\theta)$ we get

$$
\begin{gathered}
q^{\prime}(\theta)=\frac{\sin \theta}{3(3+\mu)^{2}}\left\{\frac{\left(3+\mu+e^{\lambda}(12+\mu(16+3 \mu))\right)}{e^{-\lambda}}\left(\frac{1+\cos ^{2} \theta}{\cos ^{2} \theta}\right)-3(3+\mu)^{2}\right\} \\
q^{\prime}(\theta)>0 .
\end{gathered}
$$




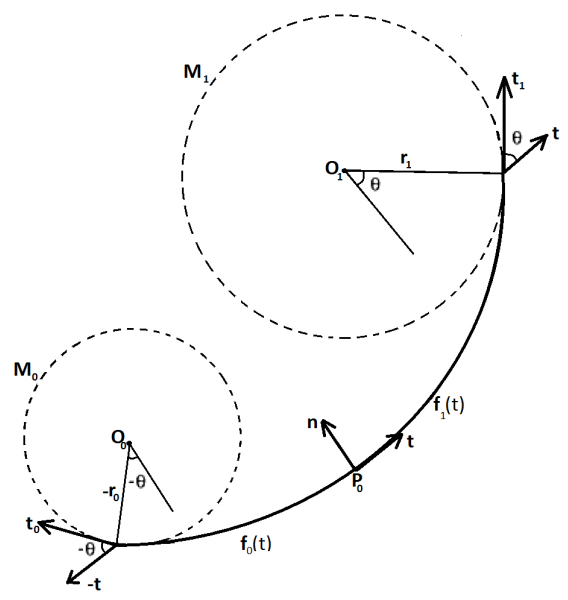

FiguRE 6. From a circle to a circle transition curve with forming a C-shaped

Therefore we have

$$
q(0)<0, \quad q(\theta)>0, \quad q^{\prime}(\theta)>0
$$

from which it follows that (3.18) has a unique solution for $0<\theta<\pi / 2$. The formula for $\sigma$ is

$$
\sigma=(\mathbf{L t}+r \sin \theta-2 a)-b \cos \theta
$$

where $\mathrm{a}$ and $\mathrm{b}$ are given in (3.2) and (3.3).

Theorem 3.3. Let $M_{0}$ and $M_{1}$ be two circles centered at $\mathbf{O}_{0}, \mathbf{O}_{1}$ with radii $r_{0}<0$, $r_{1}>0$ and

$$
|| r_{1}|-| r_{0}||=\left|r_{1}+r_{0}\right|<\left\|\mathbf{O}_{1}-\mathbf{O}_{0}\right\| .
$$

Here, $r_{0}<0$ is used to indicate direction. Therefore one circle does not enclose the other. Thus the two circles can be joined by a pair of $\lambda \mu$-Bezier like spirals forming a $C$-shaped curve such that all points of contact are $G^{2}$ as shown in Fig. (6).

Proof. Let $\mathbf{f}_{\mathbf{0}}(t ; \lambda, \mu)$ and $\mathbf{f}_{1}(t ; \lambda, \mu)$ be two $\lambda \mu$-Bezier like spirals that $M_{0}$ meets at $\mathbf{f}_{\mathbf{0}}(t ; \lambda, \mu)$ and $M_{1}$ meets at $\mathbf{f}_{1}(t ; \lambda, \mu)$. Two $\lambda \mu$-Bezier like spirals have ten degrees of freedom. We suppose that two $\lambda \mu$-Bezier like spirals meet at their beginning points $\mathbf{P}_{0}$ (where they both have zero curvature) with $G^{2}$. Suppose $-\mathbf{t}$ and $\mathbf{t}$ be the beginning unit tangent vectors of $\mathbf{f}_{\mathbf{0}}(t ; \lambda, \mu)$ and $\mathbf{f}_{1}(t ; \lambda, \mu)$ respectively. Thus three degrees of freedom disappear and remains seven degrees of freedom. Since $\mathbf{f}_{\mathbf{0}}(t ; \lambda, \mu)$ and $\mathbf{f}_{1}(t ; \lambda, \mu)$ are each free to move on the circumference of a circle, their each $G^{2}$ points of contact require three constraints on $\mathbf{f}_{\mathbf{0}}(t ; \lambda, \mu)$ and $\mathbf{f}_{1}(t ; \lambda, \mu)$. There is still one remaining degree of freedom. Let $\mathbf{t}_{0}$ and $\mathbf{t}_{1}$ be the ending unit tangent vectors of $\mathbf{f}_{\mathbf{0}}(t ; \lambda, \mu)$ and $\mathbf{f}_{1}(t ; \lambda, \mu)$, respectively. According to Theorem (3.1), the angles from $-\mathbf{t}$ to $\mathbf{t}_{0}$ and from $\mathbf{t}$ to $\mathbf{t}_{1}$ are $\theta_{0}$ and $\theta_{1}$ respectively and $-\pi / 2<\theta_{0}<0$ and $0<\theta_{1}<\pi / 2$. The remaining 
degree of freedom is used to set $\theta=-\theta_{0}=\theta_{1} ; 0<\theta<\pi / 2$. It follows now from Theorem (3.1) that

$$
\mathbf{f}_{0}(1 ; \lambda, \mu)=\mathbf{P}_{0}+2 a_{0} \mathbf{t}-b_{0} \mathbf{t}_{0}
$$

and

$$
\mathbf{f}_{1}(1 ; \lambda, \mu)=\mathbf{P}_{0}+2 a_{1} \mathbf{t}+b_{1} \mathbf{t}_{1}
$$

where

$$
\begin{aligned}
& a_{0}=\frac{r_{0} e^{-2 \lambda}\left(3+\mu+e^{\lambda}(12+\mu(16+3 \mu))\right)^{2} \sec \theta \tan \theta}{54(3+\mu)^{2}}, \\
& b_{0}=\frac{r_{0} e^{-\lambda}\left(3+\mu+e^{\lambda}(12+\mu(16+3 \mu))\right) \tan \theta}{3(3+\mu)^{2}} \\
& a_{1}=\frac{r_{1} e^{-2 \lambda}\left(3+\mu+e^{\lambda}(12+\mu(16+3 \mu))\right)^{2} \sec \theta \tan \theta}{54(3+\mu)^{2}} \\
& b_{1}=\frac{r_{1} e^{-\lambda}\left(3+\mu+e^{\lambda}(12+\mu(16+3 \mu))\right) \tan \theta}{3(3+\mu)^{2}}
\end{aligned}
$$

Hence we get

$$
\begin{aligned}
\mathbf{f}_{1}(1)-\mathbf{f}_{0}(1) & =\frac{\left(r_{1}-r_{0}\right)\left(3+\mu+e^{\lambda}(12+\mu(16+3 \mu))\right)^{2} \tan \theta}{27 e^{2 \lambda}(3+\mu)^{2} \cos \theta} \mathbf{t} \\
& +\frac{\left(3+\mu+e^{\lambda}(12+\mu(16+3 \mu)) \tan \theta\right.}{3 e^{\lambda}(3+\mu)^{2}}\left(r_{1} \mathbf{t}_{1}+r_{0} \mathbf{t}_{0}\right) .
\end{aligned}
$$

We suppose that the orientation of $\mathbf{t}$ is $\mathbf{t}\left(\mathbf{O}_{1}-\mathbf{O}_{0}\right)>0$, thus we can select one of two possible solutions. If we desired, the other solution may be selected by choosing the orientation of $\mathbf{t}$ such that $\mathbf{t}\left(\mathbf{O}_{1}-\mathbf{O}_{0}\right)<0$. It now follows that

$$
\left\{\left(\mathbf{f}_{1}(1 ; \lambda, \mu)-\mathbf{O}_{1}\right)-\left(\mathbf{f}_{0}(1 ; \lambda, \mu)-\mathbf{O}_{0}\right)\right\} \mathbf{n}=-\left(r_{1}+r_{0}\right) \cos \theta
$$

and

$$
\left\{\left(\mathbf{f}_{1}(1 ; \lambda, \mu)-\mathbf{O}_{1}\right)-\left(\mathbf{f}_{0}(1 ; \lambda, \mu)-\mathbf{O}_{0}\right)\right\} \mathbf{t}=\left(r_{1}-r_{0}\right) \sin \theta
$$

where

$$
\begin{aligned}
& \mathbf{t}_{1} \mathbf{t}=-\mathbf{t}_{0} \mathbf{t}=\cos \theta \\
& \mathbf{t}_{1} \mathbf{n}=\mathbf{t}_{0} \mathbf{n}=\sin \theta
\end{aligned}
$$

It follows from (3.25) and (3.28) that

$$
\left\{\mathbf{f}_{1}(1)-\mathbf{f}_{0}(1)\right\} \mathbf{n}=\frac{e^{-\lambda}\left(3+\mu+e^{\lambda}(12+\mu(16+3 \mu))\right) \tan \theta \sin \theta}{3(3+\mu)^{2}}\left(r_{1}+r_{0}\right)
$$

and

$$
\left\{\mathbf{f}_{1}(1)-\mathbf{f}_{0}(1)\right\} \mathbf{t}=\frac{\left(r_{1}-r_{0}\right)\left(3+\mu+e^{\lambda}(12+\mu(16+3 \mu))\right)}{e^{\lambda} 3(3+\mu)^{2} \cot \theta}\left(\frac{3+\mu+e^{\lambda}(12+\mu(16+3 \mu))}{9 e^{\lambda} \cos \theta}+\cos \theta\right)
$$

which, upon substitution into (3.26) and (3.27) and subsequent rearrangement, we obtain

$$
\begin{aligned}
& \left\{\left(\mathbf{O}_{1}-\mathbf{O}_{0}\right)\right\} \mathbf{n}=\left(r_{1}+r_{0}\right) g_{1}(\theta), \\
& \left\{\left(\mathbf{O}_{1}-\mathbf{O}_{0}\right)\right\} \mathbf{t}=\left(r_{1}-r_{0}\right) g_{2}(\theta) .
\end{aligned}
$$

Hence we derive

$$
\left\{\left(\mathbf{O}_{1}-\mathbf{O}_{0}\right)\right\} \mathbf{n}=\left\{\left(\mathbf{f}_{1}(1)-\mathbf{f}_{0}(1)\right)\right\} \mathbf{n}+\left(r_{1}+r_{0}\right) \cos \theta=\left(r_{1}+r_{0}\right) g_{1}(\theta) .
$$


Therefore we get

$$
\begin{gathered}
\left(r_{1}+r_{0}\right) g_{1}(\theta)=\frac{e^{-\lambda}\left(3+\mu+e^{\lambda}(12+\mu(16+3 \mu))\right) \tan \theta \sin \theta}{3(3+\mu)^{2}}\left(r_{1}+r_{0}\right)+\left(r_{1}+r_{0}\right) \cos \theta, \\
g_{1}(\theta)=\frac{e^{-\lambda}\left(3+\mu+e^{\lambda}(12+\mu(16+3 \mu))\right) \tan \theta \sin \theta}{3(3+\mu)^{2}}+\cos \theta .
\end{gathered}
$$

In a similar way, we have

$$
\left\{\left(\mathbf{O}_{1}-\mathbf{O}_{0}\right)\right\} \mathbf{t}=\left\{\left(\mathbf{f}_{1}(1)-\mathbf{f}_{0}(1)\right)\right\} \mathbf{t}-\left(r_{1}-r_{0}\right) \sin \theta=\left(r_{1}-r_{0}\right) g_{2}(\theta) .
$$

Therefore, we get

$$
\begin{aligned}
\left(r_{1}-r_{0}\right) g_{2}(\theta) & =\frac{\left(r_{1}-r_{0}\right)\left(3+\mu+e^{\lambda}(12+\mu(16+3 \mu))\right)}{3 \cot \theta(3+\mu)^{2} e^{\lambda}}\left(\frac{3+\mu+e^{\lambda}(12+\mu(16+3 \mu))}{9 e^{\lambda} \cos \theta}+\cos \theta\right) \\
& -\left(r_{1}-r_{0}\right) \sin \theta,
\end{aligned}
$$

or

$$
\begin{aligned}
g_{2}(\theta)=\frac{\left(3+\mu+e^{\lambda}(12+\mu(16+3 \mu))\right) \tan \theta}{3(3+\mu)^{2} e^{\lambda}} & \left(\frac{3+\mu+e^{\lambda}(12+\mu(16+3 \mu))}{9 e^{\lambda} \cos \theta}+\cos \theta\right)-\sin \theta .
\end{aligned}
$$

From (3.31) and (3.32), the pair of $\lambda \mu$-Bezier like spirals is obtained by solution of

$$
\left(r_{1}+r_{0}\right)^{2}\left\{g_{1}(\theta)\right\}^{2}+\left(r_{1}-r_{0}\right)^{2}\left\{g_{2}(\theta)\right\}^{2}=\left\|\mathbf{O}_{1}-\mathbf{O}_{0}\right\|^{2} .
$$

Set

$$
q(\theta)=\left(r_{1}+r_{0}\right)^{2}\left\{g_{1}(\theta)\right\}^{2}+\left(r_{1}-r_{0}\right)^{2}\left\{g_{2}(\theta)\right\}^{2}-\left\|\mathbf{O}_{1}-\mathbf{O}_{0}\right\|^{2},
$$

then it follows that

$$
\begin{aligned}
& g_{1}(0)= \frac{e^{-\lambda}\left(3+\mu+e^{\lambda}(12+\mu(16+3 \mu))\right) \tan 0 \sin 0}{3(3+\mu)^{2}}+\cos 0=1, \\
& g_{2}(0)= \frac{\left(3+\mu+e^{\lambda}(12+\mu(16+3 \mu))\right) \tan 0}{3(3+\mu)^{2} e^{\lambda}} \\
&\left(\frac{\left(3+\mu+e^{\lambda}(12+\mu(16+3 \mu))\right)}{9 e^{\lambda} \cos 0}+\cos 0\right)-\sin 0=0 \\
& q(0)=\left(r_{1}+r_{0}\right)^{2}-\left\|\mathbf{O}_{1}-\mathbf{O}_{0}\right\|^{2} .
\end{aligned}
$$

From the requirement in theorem

$$
\begin{gathered}
\left|r_{1}-r_{0}\right|<\left\|\mathbf{O}_{1}-\mathbf{O}_{1}\right\| \Rightarrow\left|r_{1}-r_{0}\right|^{2}<\left\|\mathbf{O}_{1}-\mathbf{O}_{1}\right\|^{2} \Rightarrow q(0)<0 \\
q(\theta \rightarrow \pi / 2) \rightarrow \infty>0
\end{gathered}
$$

and

$$
q^{\prime}(\theta)=2\left(r_{1}+r_{0}\right)^{2} g_{1}(\theta) g_{1}^{\prime}(\theta)+2\left(r_{1}-r_{0}\right)^{2} g_{2}(\theta) g_{2}(\theta)
$$




$$
\begin{gathered}
g_{1}(\theta)=\frac{\left(3+\mu+e^{\lambda}(12+\mu(16+3 \mu))\right) \tan \theta \sin \theta}{3 e^{\lambda}(3+\mu)^{2}}+\cos \theta=w_{1}(\lambda, \mu) \tan \theta \sin \theta+\cos \theta, \\
g_{1}^{\prime}(\theta)=w_{1}(\lambda, \mu)(\tan \theta \sin \theta)^{\prime}+(\cos \theta)^{\prime}=w_{1}(\lambda, \mu)(\sin \theta+\sec \theta \tan \theta)-\sin \theta \\
\theta \rightarrow \pi / 2, \sin \theta \rightarrow 1, \sec \theta \rightarrow \infty, \tan \theta \rightarrow \infty, \quad g_{1}^{\prime}(\theta) \rightarrow \infty \\
g_{2}(\theta)=\frac{\left(3+\mu+e^{\lambda}(12+\mu(16+3 \mu))\right) \tan \theta}{e^{\lambda} 3(3+\mu)^{2}}\left(\frac{\left(3+\mu+e^{\lambda}(12+\mu(16+3 \mu))\right)}{e^{\lambda} 9 \cos \theta}+\cos \theta\right)-\sin \theta, \\
g_{2}(\theta)=w_{2}(\lambda, \mu) \tan \theta \sec \theta+w_{1}(\lambda, \mu) \sin \theta-\sin \theta, \\
g_{2}^{\prime}(\theta)=w_{2}(\lambda, \mu)(\tan \theta \sec \theta)^{\prime}+\left(w_{1}(\lambda, \mu)-1\right)(\sin \theta)^{\prime} \\
=w_{2}(\lambda, \mu)\left(\sec \theta\left(\tan { }^{2} \theta+\sec ^{2} \theta\right)\right)+\left(w_{1}(\lambda, \mu)-1\right) \cos \theta \\
\theta \rightarrow \pi / 2 \quad \sec \theta \rightarrow \infty, \tan \theta \rightarrow \infty, \cos \theta \rightarrow 0, \quad g_{2}^{\prime}(\theta) \rightarrow \infty
\end{gathered}
$$

Thus, we get

$$
\begin{aligned}
q^{\prime}(\theta) & >0, \\
q(0)<0, \quad q(\theta) & >0, \quad q^{\prime}(\theta)>0 .
\end{aligned}
$$

Hence (3.33) has a unique solution for $0<\theta<\pi / 2$ and $\left|r_{1}+r_{0}\right|<\left\|\mathbf{O}_{1}-\mathbf{O}_{0}\right\|$. The $\lambda \mu$-Bezier like spirals are obtained by solving (3.33) for $\theta$, determining $\mathbf{t}, \mathbf{n}, \mathbf{t}_{0}$ and $\mathbf{t}_{1}$ from (3.31), (3.32) and (3.28), and then applying Theorem (3.1).

The following theorem can be obtained in a similar way.

Theorem 3.4. Let two circles be $M_{0}, M_{1}$ centered at $\mathbf{O}_{0}, \mathbf{O}_{1}$ with radii $r_{0}, r_{1}>0$. (This selects one of two possible solutions; for the other solution, $r_{0}, r_{1}<0$ ). If $\left|r_{1}+r_{0}\right|<\left\|\mathbf{O}_{1}-\mathbf{O}_{0}\right\|$ then the two circles can be joined by a pair of $\lambda \mu-$ Bezier like spirals forming an $S$-shaped curve such that all points of contact are $G^{2}$ as shown in Fig. (7).

The following theorem gives the result for the transition from one line to another.

Theorem 3.5. Let three points be $\mathbf{F}_{0}, \mathbf{F}_{1}$ and $\mathbf{F}$. Suppose that

$$
\mathbf{t}_{0}=\frac{\mathbf{F}-\mathbf{F}_{0}}{\left\|\mathbf{F}-\mathbf{F}_{0}\right\|}, \mathbf{t}_{1}=\frac{\mathbf{F}-\mathbf{F}_{1}}{\left\|\mathbf{F}-\mathbf{F}_{1}\right\|}
$$

and let $\alpha<\pi$ be the angle at $\mathbf{F}$ formed by $\mathbf{F}_{0}, \mathbf{F}$ and $\mathbf{F}_{1}$. For any value $c>0$, the pair of $\lambda \mu$-Bezier like spirals

$$
\mathbf{f}_{0}(t ; \lambda, \mu)=A_{0}(t ; \lambda) \mathbf{P}_{0}+A_{1}(t ; \lambda) \mathbf{P}_{1}+A_{2}(t ; \mu) \mathbf{P}_{2}+A_{3}(t ; \mu) \mathbf{P}_{3}
$$

and

$$
\mathbf{f}_{1}(t ; \lambda, \mu)=A_{0}(t ; \lambda) \mathbf{B}_{0}+A_{1}(t ; \lambda) \mathbf{B}_{1}+A_{2}(t ; \mu) \mathbf{B}_{2}+A_{3}(t ; \mu) \mathbf{B}_{3}
$$




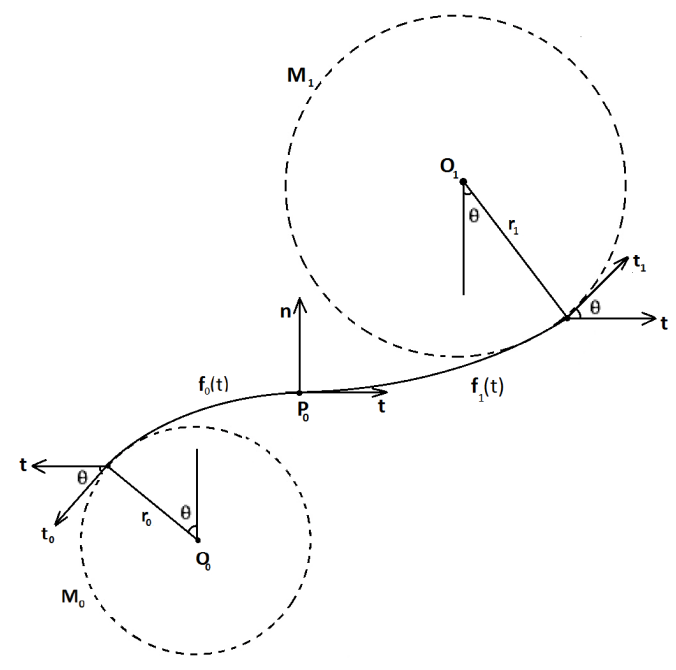

FiguRE 7. From a circle to a circle transition with an S-shaped curve.

with

$$
\begin{aligned}
A_{0}(t ; \lambda) & =(1-t)^{3} e^{-\lambda t}, \\
A_{1}(t ; \lambda) & =(1-t)^{2}\left[1+2 t-(1-t) e^{-\lambda t}\right], \\
A_{2}(t ; \mu) & =t^{2}\left[3-2 t-t e^{-\mu(1-t)}\right], \\
A_{3}(t ; \mu) & =t^{3} e^{-\mu(1-t)}, \\
\mathbf{P}_{1} & =\mathbf{P}_{0}+a \mathbf{t}_{0}, \\
\mathbf{P}_{2} & =\mathbf{P}_{1}+a \mathbf{t}_{0}=\mathbf{P}_{0}+2 a \mathbf{t}_{0}, \\
\mathbf{P}_{3} & =\mathbf{P}_{2}+b \mathbf{t}=\mathbf{P}_{0}+2 a \mathbf{t}_{0}+b \mathbf{t}, \\
\mathbf{B}_{1} & =\mathbf{B}_{0}+a \mathbf{t}_{1}, \\
\mathbf{B}_{2} & =\mathbf{B}_{1}+a \mathbf{t}_{1}=\mathbf{B}_{0}+2 a \mathbf{t}_{1}, \\
\mathbf{B}_{3} & =\mathbf{B}_{2}-b \mathbf{t}=\mathbf{B}_{0}+2 a \mathbf{t}_{1}-b \mathbf{t},
\end{aligned}
$$

where

$$
\begin{aligned}
& \theta=\frac{1}{2}(\pi-\alpha), \\
& \mathbf{t}=\frac{\mathbf{t}_{1}-\mathbf{t}_{0}}{\left\|\mathbf{t}_{1}-\mathbf{t}_{0}\right\|}, \\
& \mathbf{P}_{0}=\mathbf{F}-\sigma \mathbf{t}_{0}, \\
& \mathbf{B}_{0}=\mathbf{F}-\sigma \mathbf{t}_{1},
\end{aligned}
$$

and

$$
\begin{aligned}
\sigma & =\frac{2 a \cos \theta+b}{\cos \theta}=2 a+\frac{b}{\cos \theta}, \\
\sigma & =\frac{\left(3+\mu+e^{\lambda}(12+\mu(16+3 \mu))\right)^{2} \tan \theta}{e^{2 \lambda} 27 c(3+\mu)^{2} \cos \theta}+\frac{\left(3+\mu+e^{\lambda}(12+\mu(16+3 \mu))\right) \tan \theta}{e^{\lambda} 3 c(3+\mu)^{2} \cos \theta},
\end{aligned}
$$




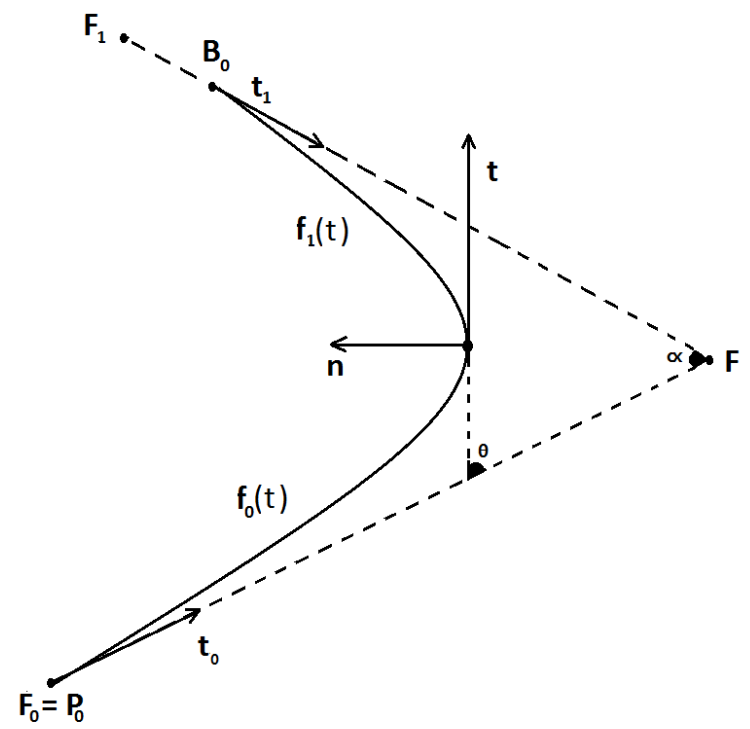

Figure 8. From a line to a line transition

$\sigma=\tan \theta \frac{\left(3+\mu+e^{\lambda}(12+\mu(16+3 \mu))\right)\left(e^{-\lambda}\left(3+\mu+e^{\lambda}(12+\mu(16+3 \mu))\right)+9\right)}{e^{\lambda} 27 c(3+\mu)^{2} \cos \theta}$

joins the two directed lines $\mathbf{F}_{0}$ and $\mathbf{F}_{1}$, and meeting at $\mathbf{P}_{3}=\mathbf{B}_{3}$, such that all points of contact are $G^{2}$ as shown in Fig. (8); the absolute value of the curvature of the two spirals at their joint is $c$.

Proof. Two $\lambda \mu$-Bezier like spirals have ten degrees of freedom. Let $\mathbf{t}$ be the unit tangent vector at $t=1$. Let $c$ be the curvature of $\mathbf{f}_{0}(t ; \lambda, \mu)$ at $t=1$. To ensure a $G^{2}$ match where $\mathbf{f}_{0}(t ; \lambda, \mu)$ and $\mathbf{f}_{1}(t ; \lambda, \mu)$ meet, from the condition

$$
\mathbf{f}_{0}(1 ; \lambda, \mu)=\mathbf{f}_{1}(1 ; \lambda, \mu)
$$

the unit tangent vector should be $-\mathbf{t}$ and the curvature of $\mathbf{f}_{1}(t ; \lambda, \mu)$ should be $-c$ at $t=1$. There are thus six remaining degrees of freedom. Since $\mathbf{f}_{0}(t ; \lambda, \mu)$ and $\mathbf{f}_{1}(t ; \lambda, \mu)$ are each free to move along a line, i.e.,

$$
\begin{aligned}
& \mathbf{P}_{0}=\mathbf{F}-\sigma_{0} \mathbf{t}_{0}, \\
& \mathbf{B}_{0}=\mathbf{F}-\sigma_{1} \mathbf{t}_{1}
\end{aligned}
$$

and since both $\mathbf{f}_{0}(t ; \lambda, \mu)$ and $\mathbf{f}_{1}(t ; \lambda, \mu)$ have zero curvature at their beginning points because of the $G^{2}$ requirements at these points of contact each impose two constraints on $\mathbf{f}_{0}(t ; \lambda, \mu)$ and $\mathbf{f}_{1}(t ; \lambda, \mu)$. There are still two remaining degrees of freedom. The beginning unit tangent vectors of $\mathbf{f}_{0}(t ; \lambda, \mu)$ and $\mathbf{f}_{1}(t ; \lambda, \mu)$ are $\mathbf{t}_{0}$ and $\mathbf{t}_{1}$ respectively. According to Theorem (3.1), the angles from $\mathbf{t}_{0}$ to $\mathbf{t}$ and from $\mathbf{t}_{1}$ to $-\mathbf{t}$ are $\theta_{0}$ and $\theta_{1}$ respectively where $0<\theta_{0}<\pi / 2$ and $-\pi / 2<\theta_{1}<0$. One of the remaining degrees of freedom is used to set $\theta=\theta_{0}=-\theta_{1}$, hence 


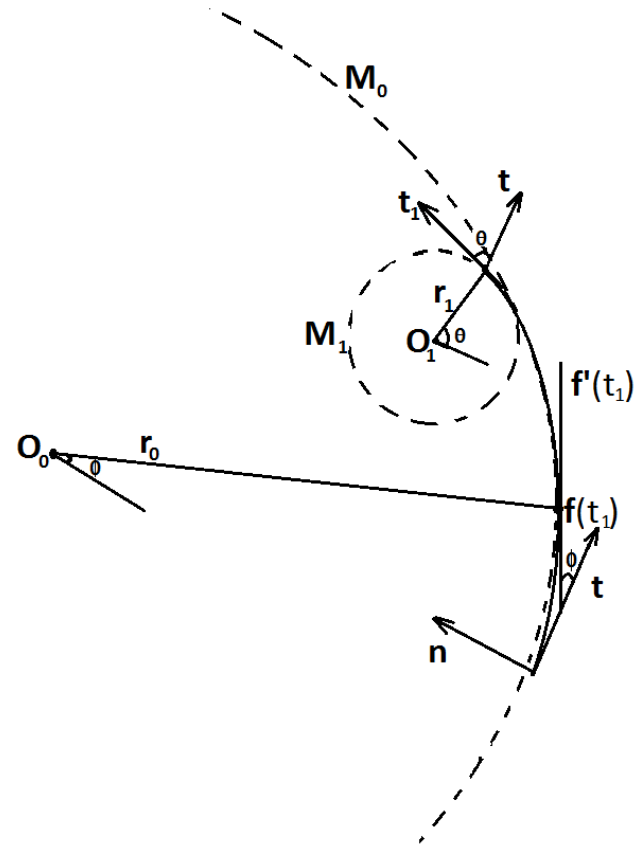

Figure 9. Circle to circle transition with a single spiral.

$\theta=\frac{1}{2}(\pi-\alpha)$ and

$$
\mathbf{t}=\frac{\mathbf{t}_{1}-\mathbf{t}_{0}}{\left\|\mathbf{t}_{1}-\mathbf{t}_{0}\right\|}
$$

Thus we simplify subsequent algebraic expressions and balance the angle of the tangent of the two $\lambda \mu$-Bezier like spirals. The last remaining degree of freedom $c$ is for the curve designer to use as a shape parameter by choosing a value for it. If follows now from (3.40) in conjunction with (3.34) to (3.38), (3.41) and (3.42) that

$$
\begin{gathered}
\mathbf{P}_{0}=\mathbf{F}-\sigma_{0} \mathbf{t}_{0} \\
\mathbf{B}_{0}=\mathbf{F}-\sigma_{1} \mathbf{t}_{1} \\
\mathbf{P}_{0}-\mathbf{B}_{0}=\sigma_{1} \mathbf{t}_{1}-\sigma_{0} \mathbf{t}_{0} \\
\mathbf{f}_{\mathbf{0}}(1 ; \lambda, \mu)=\mathbf{f}_{1}(1 ; \lambda, \mu) \Rightarrow \mathbf{P}_{0}-\mathbf{B}_{0}=2 a\left(\mathbf{t}_{1}-\mathbf{t}_{0}\right)-2 b \mathbf{t},
\end{gathered}
$$

Since (3.43) is equal to (3.44), taking the dot product of this equation and $\mathbf{n}$ and using $\mathbf{t}_{0} \mathbf{n}=\mathbf{t}_{1} \mathbf{n}=-\sin \theta$, we obtain

$$
\sigma_{0}=\sigma_{1}=\sigma
$$

Similarly, taking the dot product of this equation and $\mathbf{t}$ and using $\mathbf{t}_{0} \mathbf{t}=-\mathbf{t}_{1} \mathbf{t}=$ $\cos \theta$, we obtain

$$
\sigma=\frac{2 a \cos \theta+b}{\cos \theta}=2 a+\frac{b}{\cos \theta}
$$


For the fifth case (from a circle to a circle, one circle inside the other), a transition curve with six degrees of freedom is required for $G^{2}$ contact with the circles. The beginning point of the $\lambda \mu$-Bezier like spiral, $\mathbf{P}_{0}$, cannot be used as a point of contact because the curvature there is 0 . An additional degree of freedom is the parameter value of the unknown point of contact of the larger circle with the arc of the $\lambda \mu$-Bezier like spiral. The radii are the same sign and are supposed to be positive. An analogous analysis can be done for negative radii. Consider two circles $M_{0}, M_{1}$ centered at $\mathbf{O}_{0}, \mathbf{O}_{1}$ with radii $r_{0}, r_{1}>0$ and such that $M_{1}$ is completely contained inside $M_{0}$. It is desirable to join the two circles by a single $\lambda \mu$-Bezier like spiral such that both points of contact are $G^{2}$ as shown in Fig.(9). Let the $\lambda \mu$-Bezier like spiral as defined in Theorem (3.1) meets $M_{0}$ at $t=t_{1}$ and $M_{1}$ at $t=1$ respectively. Let the angle from $\mathbf{t}$ to the unit tangent at $t=t_{1}$ be $\phi$. It follows from (3.14)

$$
\kappa\left(t_{1}\right)=\frac{\left\{x^{\prime}\left(t_{1}\right) y^{\prime \prime}\left(t_{1}\right)-x^{\prime \prime}\left(t_{1}\right) y^{\prime}\left(t_{1}\right)\right\}}{\left\{\left(x^{\prime}\left(t_{1}\right)\right)^{2}+\left(y^{\prime}\left(t_{1}\right)\right)^{2}\right\}^{3 / 2}}=\frac{r_{1}}{r_{0}} .
$$

Observe that if $\theta$ is known then (3.45), it can be solved uniquely for $t_{1}$ in $(0,1)$ by Theorem (3.1), the derivative with respect to $t_{1}$ of the left-hand side does not change sign on $(0,1)$; for $t_{1}=0$, the left-hand side is zero $\left(<\frac{r_{1}}{r_{0}}\right)$, and for $t_{1}=1$, the left-hand side equals unity $\left(>\frac{r_{1}}{r_{0}}\right)$. By taking the dot product of $\mathbf{f}^{\prime}(t ; \lambda, \mu)$ at $t=t_{1}$ with the unit normal vector, $\mathbf{n}$ and unit tangent vector, $\mathbf{t}$, of $\mathbf{f}(t ; \lambda, \mu)$ at $t=0$, it follows from (3.9)

$$
\begin{gathered}
\mathbf{f}^{\prime}\left(t_{1}\right) \mathbf{t}=\frac{r_{1}\left(3+\mu+e^{\lambda}(12+\mu(16+3 \mu))\right)^{2} \tan \theta e^{-t_{1} \lambda}\left(1-t_{1}\right)^{2}\left(3+\left(1-t_{1}\right) \lambda\right)}{e^{2 \lambda} 54(3+\mu)^{2} \cos \theta} \\
+\frac{r_{1}\left(3+\mu+e^{\lambda}(12+\mu(16+3 \mu)) \tan \theta t_{1}^{2}\left(3+\mu t_{1}\right) \cos \theta\right.}{e^{\lambda} 3(3+\mu)^{2} e^{\mu\left(1-t_{1}\right)}} \\
\mathbf{f}^{\prime}\left(t_{1}\right) \mathbf{n}=t_{1}^{2} \frac{r_{1}\left(3+\mu+e^{\lambda}(12+\mu(16+3 \mu))\right)\left(3+\mu t_{1}\right)}{3 e^{\lambda+\mu\left(1-t_{1}\right)}(3+\mu)^{2}} \sin \theta \tan \theta \\
\mathbf{f}^{\prime}\left(t_{1}\right)=\cos \phi \mathbf{t}+\sin \phi \mathbf{n} \\
\mathbf{f}^{\prime}\left(t_{1}\right) \mathbf{t}=\cos \phi \mathbf{t} \mathbf{t}+\sin \phi \mathbf{n t}=\cos \phi \\
\mathbf{f}^{\prime}\left(t_{1}\right) \mathbf{n}=\cos \phi \mathbf{t n}+\sin \phi \mathbf{n n}=\sin \phi \\
\mathbf{f}^{\prime}\left(t_{1}\right) \mathbf{t}=\cos \phi=a \cdot e^{-t_{1} \lambda}\left(1-t_{1}\right)^{2}\left(3+\left(1-t_{1}\right) \lambda\right)+b \cos \theta e^{-\mu\left(1-t_{1}\right)} t_{1}^{2}\left(3+\mu t_{1}\right) \\
\mathbf{f}^{\prime}\left(t_{1}\right) \mathbf{n}=\sin \phi=t_{1}^{2} \frac{r_{1}\left(3+\mu+e^{\lambda}(12+\mu(16+3 \mu))\right)\left(3+\mu t_{1}\right)}{3 e^{\lambda+\mu\left(1-t_{1}\right)}(3+\mu)^{2}} \sin \theta \tan \theta
\end{gathered}
$$

From (3.9), we have

and

$$
\frac{\mathbf{f}^{\prime}\left(t_{1}\right) \mathbf{n}}{\mathbf{f}^{\prime}\left(t_{1}\right) \mathbf{t}}=\frac{\sin \phi}{\cos \phi}=\tan \phi
$$

$$
\tan \phi=\frac{18 t_{1}^{2}\left(3+\mu t_{1}\right) e^{\mu\left(t_{1}-t\right)} e^{\lambda\left(t_{1}+1\right)} \sin \theta \cos \theta}{Q(\lambda, \mu, \theta)}
$$

where

$$
\begin{aligned}
Q(\lambda, \mu, \theta) & =\left(3+\mu+e^{\lambda}(12+\mu(16+3 \mu))\right) e^{\mu\left(1-t_{1}\right)}\left(1-t_{1}\right)^{2}\left(3+\left(1-t_{1}\right) \lambda\right) \\
& +18 e^{\left(1+t_{1}\right) \lambda} \cos ^{2} \theta t_{1}^{2}\left(3+\mu t_{1}\right)
\end{aligned}
$$


It follows from Theorem (3.1) that

$$
\mathbf{f}(1)=\mathbf{P}_{0}+2 a \mathbf{t}+b \mathbf{t}_{1}
$$

and, writing $\mathbf{f}\left(t_{1}\right)$ in monomial form, that

$$
\mathbf{f}\left(t_{1}\right)=\mathbf{P}_{0}+a\left(A_{1}\left(t_{1} ; \lambda\right)+2 A_{2}\left(t_{1} ; \mu\right)+2 A_{3}\left(t_{1} ; \mu\right)\right) \mathbf{t}+A_{3}\left(t_{1} ; \mu\right) b \mathbf{t}_{1} .
$$

From (2.2), we obtain

$$
\begin{aligned}
\mathbf{f}\left(t_{1}\right) & =\mathbf{P}_{0}+a\left(1-e^{-\lambda t_{1}}\right) \mathbf{t}+a\left(3 e^{-\lambda t_{1}}\right) t_{1} \mathbf{t}+3 a\left(1-e^{-\lambda t_{1}}\right) t_{1}^{2} \mathbf{t} \\
& +\left(a\left(e^{-\lambda t_{1}}-2\right) \mathbf{t}+e^{-\mu\left(1-t_{1}\right)} b \mathbf{t}_{1}\right) t_{1}^{3} \\
& =\mathbf{P}_{0}+a\left\{\left(1-e^{-\lambda t_{1}}\right)+\left(3 e^{-\lambda t_{1}}\right) t_{1}+3\left(1-e^{-\lambda t_{1}}\right) t_{1}^{2}+\left(e^{-\lambda t_{1}}-2\right) t_{1}^{3}\right\} \mathbf{t} \\
& +\left(b e^{-\mu\left(1-t_{1}\right)}\right) t_{1}^{3} \mathbf{t}_{1} .
\end{aligned}
$$

Subtraction of $\mathbf{f}\left(t_{1}\right)$ from $\mathbf{f}(1)$ with subsequent rearrangement yields

$$
\begin{aligned}
\mathbf{f}(1)-\mathbf{f}\left(t_{1}\right) & =a \mathbf{t} \\
& \left\{2-\left(1-e^{-\lambda t_{1}}\right)+\left(3 e^{-\lambda t_{1}}\right) t_{1}+3\left(1-e^{-\lambda t_{1}}\right) t_{1}^{2}+\left(e^{-\lambda t_{1}}-2\right) t_{1}^{3}\right\} \\
& +b\left\{1-e^{-\mu\left(1-t_{1}\right)} t_{1}^{3}\right\} \mathbf{t}_{1}
\end{aligned}
$$

It also follows that

$$
\left\{\left(\mathbf{f}(1)-\mathbf{O}_{1}\right)-\left(\mathbf{f}\left(t_{1}\right)-\mathbf{O}_{0}\right)\right\} \mathbf{n}=-\left(r_{1} \cos \theta-r_{0} \cos \phi\right)
$$

and

$$
\left\{\left(\mathbf{f}(1)-\mathbf{O}_{1}\right)-\left(\mathbf{f}\left(t_{1}\right)-\mathbf{O}_{0}\right)\right\} \mathbf{t}=\left(r_{1} \sin \theta-r_{0} \sin \phi\right)
$$

Taking the dot product of (3.50) with $\mathbf{t}$ and $\mathbf{n}$ with subsequent substitution into (3.51) and (3.52) and rearrangement produce

$$
\left\{\left(\mathbf{O}_{1}-\mathbf{O}_{0}\right)\right\} \mathbf{n}=b\left\{1-e^{-\mu\left(1-t_{1}\right)} t_{1}^{3}\right\} \sin \theta+r_{1} \cos \theta-r_{0} \cos \phi
$$

and

$$
\begin{aligned}
\left\{\left(\mathbf{O}_{1}-\mathbf{O}_{0}\right)\right\} \mathbf{t} & =a\left(2-\left(1-e^{-\lambda t_{1}}\right)+\left(3 e^{-\lambda t_{1}}\right) t_{1}+3\left(1-e^{-\lambda t_{1}}\right) t_{1}^{2}\right. \\
& \left.+\left(e^{-\lambda t_{1}}-2\right) t_{1}^{3}\right)+b\left\{1-e^{-\mu\left(1-t_{1}\right)} t_{1}^{3}\right\} \cos \theta-r_{1} \sin \theta+r_{0} \sin \phi
\end{aligned}
$$

Hence we get

$$
\begin{aligned}
g_{1}(\theta) & =\left\{\left(\mathbf{O}_{1}-\mathbf{O}_{0}\right)\right\} \mathbf{n}=b\left\{1-e^{-\mu\left(1-t_{1}\right)} t_{1}^{3}\right\} \sin \theta+r_{1} \cos \theta-r_{0} \cos \phi \\
g_{2}(\theta) & =\left\{\left(\mathbf{O}_{1}-\mathbf{O}_{0}\right)\right\} \mathbf{t} \\
& =a\left(2-\left(1-e^{-\lambda t_{1}}\right)+\left(3 e^{-\lambda t_{1}}\right) t_{1}+3\left(1-e^{-\lambda t_{1}}\right) t_{1}^{2}+\left(e^{-\lambda t_{1}}-2\right) t_{1}^{3}\right) \\
& +\left\{1-e^{-\mu\left(1-t_{1}\right)} t_{1}^{3}\right\} \cos \theta-r_{1} \sin \theta+r_{0} \sin \phi
\end{aligned}
$$

because of that the $\lambda \mu$-Bezier like spiral, if it exists, is obtained by solution of

$$
q(\theta)=\left\{g_{1}(\theta)\right\}^{2}+\left\{g_{2}(\theta)\right\}^{2}-\left\|\mathbf{O}_{1}-\mathbf{O}_{0}\right\|^{2}=0 .
$$

(3.45), (3.48) and (3.57) are three concurrent nonlinear equations in $t_{1}, \phi$ and $\theta$. If a solution exists, it can be found numerically by (3.57) as a nonlinear equation in a single unknown, because for any given $\theta, t_{1}$ can be found from $(3.45), \phi$ can be found from (3.48), so $q(\theta)$ can be assessed. 


\section{References}

[1] U. Bashir, M. Abbas, M. N. Awang and J. M. Ali, The quadratic trigonometric Bezier curve with single shape parameter, Journal of Basic and Applied Scientific Research 2(3) (2012), 2541-2546.

[2] U. Bashir, M. Abbas and J. M. Ali, The $G^{2}$ and $C^{2}$ rational quadratic trigonometric Bezier curve with two shape parameters with applications, Applied Mathematics and Computation 219(20) (2013), 10183-10197.

[3] L. Chu and X. M. Zeng, Constructing curves and triangular patches by beta functions, J. Comput. Appl. Math. 260 (2014), 191-200.

[4] G. Farin, Curves and surfaces for CAGD: A Practical Guide, Academic Press, 2002.

[5] Z. Habib and M. Sakai, Spiral transition curves and their applications, Scientiae Mathematicae Japonicae (2004), 251-262.

[6] A. Levent and B Sahin, Beta BÃ (C)zier curves, Appl. Comput. Math. 18 (2019), 1, 79-94.

[7] A. Levent and B. Sahin, Cubic Bezier-like transition curves with new basis functions, Proceedings of the Institute of Mathematics and Mechanics, National Academy of Sciences of Azerbaijan 44 (2018), 2, 222-228.

[8] A. Levent, B. Sahin and Z. Habib, Spiral transitions, Appl. Math. J. Chinese Univ. 33 (2018), 468-490.

[9] R. S. Millman and G. D. Parker, Elements of differential geometry, Englewood Cliffs, N.J.: Prentice-Hall, 1977.

[10] H. G. Timmer, Alternative representation for parametric cubic curves and surfaces, IPC Business Press 12, 1, 1980.

[11] D.J. Walton and D.S. Meek, A planar cubic Bezier spiral, J. Comput. Appl. Math. 72 (1996), 85-100.

[12] D. J. Walton, D. S. Meek and J. M. Ali, Planar $G^{2}$ transition curves composed of cubic Bezier spiral segments, J. Comput. Appl. Math. 157 (2003), 453-476.

[13] D. J. Walton and D. S. Meek, A further generalisation of the planar cubic Bezier spiral, Journal of Computational and Applied Mathematics 236 (2012), 2869-2882.

[14] Y. Zhu and X. Han, Curves and surfaces construction based on new basis with exponential functions, Acta Appl Math (2014), 129-183.

Asli Ayar

Department of Mathematics, Ege University, Izmir, Turkey.

E-mail address: asliayar1@gmail.com

Bayram Şahin

Department of Mathematics, Ege University, Izmir, Turkey.

E-mail address: bayram.sahin@ege.edu.tr

Received: July 12, 2020; Accepted: December 29, 2020 